MARTINELLI, S.; MONTAGNA, M.A.; PICINATO, N.C.; SILVA, F.M.A.; FERNANDES, O.A. Eficácia do indoxacarb para o controle de pragas em hortaliças. Horticultura Brasileira, Brasília, v. 21, n. 3, p. 501-505, julho-setembro 2003.

\title{
Eficácia do indoxacarb para o controle de pragas em hortaliças
}

\author{
Samuel Martinellii ${ }^{1}$; Marco Aurélio Montagna²; Nilton César Picinato ${ }^{3}$; Fábio M.A. Silva ${ }^{3}$; Odair Apareci- \\ do Fernandes ${ }^{4}$ \\ ${ }^{1 / E S A L Q / U S P, ~ 13418-900 ~ P i r a c i c a b a-S P ; ~}{ }^{2 /}$ Ecolab, R. Alvorada, 48, 04550-000 São Paulo-SP; ${ }^{3 /}$ Du Pont do Brasil S.A., C. Postal 09, \\ 13140-000 Paulínia-SP; ${ }^{4 / F C A V / U N E S P, ~ R o d . ~ P r o f . ~ P a u l o ~ D . ~ C a s t e l l a n e ~ k m ~ 5, ~ 14884-900 ~ J a b o t i c a b a l-S P ; ~ E-m a i l: ~}$ \\ oafernan@fcav.unesp.br
}

\section{RESUMO}

A eficiência do indoxacarb foi avaliada no controle dos lepidópteros-pragas em hortaliças: Plutella xylostella (Lepidoptera: Yponeumatidae), Trichoplusia ni (Lepidoptera: Noctuidae), Neoleucinodes elegantalis (Lepidoptera: Pyralidae) e Helicoverpa zea (Lepidoptera: Noctuidae). Os experimentos foram conduzidos em três locais diferentes nas culturas do repolho e tomate. Os inseticidas utilizados foram indoxacarb (no tomate de 2,4 a 6,0 g i.a. $/ 100$ $\mathrm{L}$ e no repolho de 18 a $42 \mathrm{~g}$ i.a./ha), esfenvalerate (apenas no tomate de 1,75 g i.a./100 L), metomil (no tomate na dose de 21,5 g i.a./100 $\mathrm{L}$ e no repolho a $172 \mathrm{~g}$ i.a./ha), deltametrina (apenas no repolho na dose de $6 \mathrm{~g}$ i.a./ha) e triflumuron (apenas no tomate na dose de $15 \mathrm{~g}$ i.a./100 L). Adotou-se o delineamento estatístico em blocos ao acaso com quatro repetições em todos os experimentos. Todas as doses avaliadas do produto indoxacarb apresentaram excelente desempenho no controle de P. xylostella e T. ni. Assim, a menor dose avaliada (18 g i.a./ha) pode ser recomendada para o controle dessas pragas. Todos os produtos avaliados alcançaram níveis de eficiência maiores que $80 \%$ no controle de $H$. zea na cultura do tomate. Indoxacarb foi tão eficiente quanto o produto padrão adotado (triflumuron). N. elegantalis foi eficientemente controlada pelos inseticidas avaliados após nove aplicações, sendo que indoxacarb pode ser recomendado na menor dose utilizada $(2,4$ g i.a./100 L).

Palavras-chave: Plutella xylostella, Trichoplusia ni, Neoleucinodes elegantalis, Helicoverpa zea, controle químico, hortaliças.

\section{ABSTRACT}

\section{Efficacy of indoxacarb in the control of vegetable pests}

The efficacy of indoxacarb was evaluated for controlling lepidopterous pests on vegetables: Plutella xylostella (Lepidoptera: Yponeumatidae), Trichoplusia ni (Lepidoptera: Noctuidae), Neoleucinodes elegantalis (Lepidoptera: Pyralidae), Helicoverpa zea (Lepidoptera: Noctuidae). The experiments were carried out at three different sites on cabbage and tomato crops. The utilized insecticides were indoxacarb (for tomato from 2.4 to $6.0 \mathrm{~g}$ a.i. $/ 100 \mathrm{~L}$ and for cabbage from 18 to $42 \mathrm{~g}$ i.a./ha), esfenvalerate (only for tomato at $1.75 \mathrm{~g}$ a.i./100 L), methomyl (for tomato at $21.5 \mathrm{~g}$ a.i./100 L and for cabbage at $172 \mathrm{~g}$ a.i./ha), deltamethrin (only for cabbage at $6 \mathrm{~g}$ a.i./ ha) and triflumuron (only for tomato at $15 \mathrm{~g}$ a.i./100 L). The experiments were designed as randomized blocks with four replications. The results showed that all doses of indoxacarb presented excellent performance controlling P. xylostella and T. ni on cabbage. Therefore, the lowest tested dose should be used (18 g a.i./100 L). All products reached levels of efficacy higher than $80 \%$ for controlling $H$. zea on tomato crop. Indoxacarb was as efficient as the standard product triflumuron. $N$. elegantalis was efficiently controlled by the tested insecticides after nine applications and indoxacarb could be recommended at the lowest utilized dose $(2.4 \mathrm{~g}$ a.i./100 L).

Keywords: Plutella xylostella, Trichoplusia ni, Neoleucinodes elegantalis, Helicoverpa zea, chemical control, vegetables.

\section{(Recebido para publicação em 15 de maio de 2002 e aceito em 30 de abril de 2003)}

A produção brasileira de olerícolas encontra-se em pleno crescimento, estimulada pela mudança no hábito alimentar do consumidor, que passou a consumir hortaliças com maior freqüência e a exigir produtos de melhor qualidade. Os sinais de tal crescimento podem ser notados devido ao aumento do consumo de hortaliças in natura e préprocessados (AGRIANUAL, 2000).

A incidência de pragas em campos de produção de hortaliças contribui para a menor produtividade e conseqüente queda, que pode superar em $60 \%$ da produção total em casos como tomate e repolho (Barbosa \& França, 1980; Castelo Branco \& Guimarães, 1990). Assim, as hortaliças apresentam grande demanda pela aplicação de defensivos agrícolas (Nakano, 1999), pois o controle quí- mico é a principal (ou a única) tática de controle de pragas adotada. Entretanto, o controle pode ser comprometido devido aos efeitos colaterais causados pelo uso exclusivo de inseticidas. A descoberta e utilização de inseticidas que apresentem novos modos de ação podem contribuir consideravelmente para a substituição de produtos tradicionalmente utilizados (Wing et al., 2000) e favorecer o manejo da resistência de insetos a inseticidas por meio da rotação de compostos dentro de um programa de manejo integrado de pragas em hortaliças. Visando o controle da traçadas-crucíferas, Plutella xylostella. França \& Medeiros (1998) verificaram que a combinação dos produtos deltametrina + abamectina resultou em maior quantidade de cabeças comercializáveis de repolho, superando os resultados obtidos com o tratamento padrão adotado (Bacillus thuringiensis). Por outro lado, Monnerat et al. (2000) observaram que tanto abamectina (Vertimec $\AA$ ) como o inseticida biológico à base de $B$. thuringiensis $($ Biobit $\AA$ ) causaram redução da população da traça-dascrucíferas, sendo que os tratamentos apresentaram índices semelhantes de produção de cabeças comercializáveis de repolho. De acordo com Reis \& Souza (1996), os inseticidas abamectina e triflumuron foram tão eficientes quanto o padrão adotado (permetrina) no controle da broca-pequena, Neoleucinodes elegantalis, em tomate estaqueado.

$\mathrm{O}$ produto indoxacarb, novo inseticida do grupo químico das oxidiazinas, apresenta atividade sobre insetos da or- 
dem Lepidoptera, cuja alimentação é inibida após o tratamento (Pluschkell et al., 1998; Wing et al., 1998). Indoxacarb é compatível ambientalmente e seguro para organismos não alvos do controle químico, como o parasitóide Aphidius rhipalisiphi (Hymenoptera: Aphididae), o ácaro predador Typhlodromus pyri (Acari: Phitoseiidae) e o predador Episyrphus balteatus (Diptera: Syrphidae) (Harder et al., 1996; Wing et al., 1998). Além da baixa toxicidade aos organismos não-alvo, o indoxacarb apresenta taxas de dissipação ambiental muito favoráveis ao seu uso como inseticida na agricultura (McCann et al. 2001). O efeito inseticida ocorre após a bioativação do indoxacarb através da ação de enzimas (provavelmente esterases/amidases) presentes no inseto e que resultam na formação do metabólito JT333 (N-decarbomethoxyllated) que causa bloqueio dos canais de sódio. A metabolização do indoxacarb em JT333 relaciona-se com o surgimento de sintomas de neurointoxicação, os quais compreendem desde pequenas convulsões até a paralisia do inseto seguida de morte (Wing et al., 2000).

O objetivo deste trabalho foi avaliar a eficiência do indoxacarb no controle das principais pragas de hortaliças: a traça-das-crucíferas, $P$. xylostella (Lepidoptera: Yponomeutidae); a lagarta mede-palmo, Trichoplusia ni (Lepidoptera: Noctuidae); a broca-pequena, $N$. elegantalis (Lepidoptera: Pyralidae) e a broca-grande, Helicoverpa zea (Lepidopteta: Noctuidae).

\section{MATERIAL E MÉTODOS}

\section{Experimento 1}

O estudo foi desenvolvido na cultura de repolho, híbrido Kenzan, cultivado no espaçamento de $90 \mathrm{~cm}$ entre ruas e $50 \mathrm{~cm}$ entre plantas, em São João da Boa Vista, SP. Adotou-se o delineamento experimental de blocos casualizados com oito tratamentos (indoxacarb [18; 24; 30; 36 e 42 g i.a./ha], deltametrina [6 g i.a./ha], esfenvalerate [172 g i.a./ ha] e testemunha sem aplicação de inseticidas) e quatro repetições, com cada parcela medindo 4,5 x 5,0 $\mathrm{m}$ de área total. As aplicações dos produtos inicia- ram-se trinta dias após o transplante das mudas, quando a cultura apresentava infestação natural da traça-dascrucíferas. Os produtos foram aplicados em intervalos semanais de 11/03 a 10/ 04/1997, perfazendo um total de cinco aplicações. A pulverização dos produtos foi realizada com o auxílio de pulverizador costal $\left(\mathrm{CO}_{2}\right)$ dotado de barra de aplicação (2,5 $\mathrm{m}$ de comprimento) com cinco bicos XR 110-04 (pressão de trabalho de $60 \mathrm{lb} / \mathrm{pol}^{2}$ ), calibrado para o consumo de $800 \mathrm{~L}$ de calda/ha. Adicionou-se espalhante adesivo (Agral $\left.{ }^{\circledR}\right)$ à calda de pulverização $(30 \mathrm{~mL} / 100 \mathrm{~L}) \mathrm{em}$ todos os tratamentos.

As avaliações, num total de quatro, constituíram-se na observação de dez plantas tomadas ao acaso na porção central de cada parcela e aferição dos parâmetros: número de lagartas da traça-das-crucíferas por planta e nota de dano foliar decorrente da presença da lagarta nas plantas. As notas de dano basearam-se na escala: nota $0,5=$ planta não apresentando danos nas folhas laterais da cabeça; nota 5 = planta apresentando folhas laterais raspadas ou com pequenos furos; nota $10=$ planta apresentando folhas laterais furadas; nota 20 = planta apresentando folhas laterais furadas e cabeça com pequenos furos; nota $30=$ planta apresentando folhas laterais com furos grandes e cabeça com pequenos furos; nota $40=$ planta apresentando folhas laterais com furos grandes e cabeça começando a apresentar furos grandes; nota 50 = planta apresentando folhas laterais e cabeça com vários furos pequenos e grandes; nota 70 = planta apresentando folhas laterais e cabeça com vários furos grandes; nota $90=$ planta apresentando folhas laterais e cabeça com folhas praticamente destruídas; nota 100 = planta apresentando-se totalmente destruída. As três primeiras avaliações foram realizadas sete dias após a $3^{\mathrm{a}}, 4^{\mathrm{a}}$ e $5^{\mathrm{a}}$ aplicação. $\mathrm{Na}$ $3^{\text {a }}$ avaliação realizou-se apenas a observação dos danos causados. Por ocasião da $4^{\mathrm{a}}$ avaliação realizou-se a colheita de dez plantas e observou-se a presença de lagartas nas mesmas. Ainda, atribuiu-se nota de dano causado pela traça-dascrucíferas às partes superior e inferior das cabeças de repolho separadamente. Nesta ocasião ( $4^{\mathrm{a}}$ avaliação) observou- se e registrou-se a presença de lagartas de $T$. $n i$, as quais até então não haviam sido detectadas. Realizou-se a análise de variância dos dados e as médias dos tratamentos foram comparadas através do teste de Tukey ao nível de 5\% de probabilidade, utilizando-se PROC GLM (SAS Institute, Cary, NC, USA).

\section{Experimento II}

Foi realizado em área de plantio de tomate estaqueado em Mogi-Guaçu, SP. No plantio utilizaram-se plantas do híbrido Santa Clara, seguindo-se o espaçamento de $1 \mathrm{~m}$ entre linhas e 0,70 $\mathrm{m}$ entre plantas. $\mathrm{O}$ plano experimental adotado foi em blocos casualizados com sete tratamentos e quatro repetições, sendo cada parcela constituída de 20 plantas (duas linhas de dez plantas). Os tratamentos adotados para controle da broca-pequena foram indoxacarb nas doses de 2,4; 3,6; 4,8 e 6,0 g i.a./100 L, esfenvalerate na dose de $1,75 \mathrm{~g}$ i.a. $/ 100$ $\mathrm{L}$, metomil na dose de $21,5 \mathrm{~g}$ i.a./100 L e a testemunha sem aplicação de inseticidas. Essas doses foram baseadas no uso de $1.000 \mathrm{~L}$ de calda/ha.

Os tratamentos foram aplicados inicialmente quando as plantas encontravam-se no estágio de florescimento e, portanto, foram realizados preventivamente. Os tratamentos foram aplicados com o auxílio de pulverizador costal $\left(\mathrm{CO}_{2}\right)$, dotado de barra de aplicação de lança com 3 bicos (Yamaro D6), pressão de trabalho de 50 a $90 \mathrm{lb} / \mathrm{pol}^{2}$, calibrado para o consumo de $1.000 \mathrm{~L}$ de calda/ha. As aplicações foram realizadas em intervalos semanais de 20/03 a 16/05/97.

As avaliações foram realizadas cinco dias após a $5^{\mathrm{a}}$ aplicação e quatro dias após a $9^{a}$ aplicação. Foi observada a presença de danos de N. elegantalis em cinco frutos de cada uma das cinco plantas amostradas aleatoriamente na porção central de cada parcela, totalizando 25 frutos por parcela. Posteriormente, calculou-se a porcentagem de frutos brocados por parcela e a eficiência de controle de cada tratamento utilizando-se a fórmula de Abbott (Abbott, 1925). Realizou-se a análise de variância dos dados e as médias obtidas foram comparadas através do teste de Tukey ao nível de 5\% de probabilidade utilizando-se PROC GLM (SAS Institute, Cary, NC, USA). 
Tabela 1. Número médio de lagartas de traça-das-crucíferas, Plutella xylostella (Lepidoptera: Yponomeutidae) e lagarta-mede-palmo, Trichoplusia ni (Lepidoptera: Noctuidae) e nota média de dano causado pela traça-das crucíferas, em dez plantas de repolho, submetidas a diferentes tratamentos inseticidas. São João da Boa Vista (SP), FCAV/UNESP, 1997.

\begin{tabular}{|c|c|c|c|c|c|c|c|c|c|}
\hline \multirow{3}{*}{ Tratamento } & \multirow{3}{*}{$\begin{array}{c}\text { Dose } \\
\text { (g i.a./ha) }\end{array}$} & \multicolumn{4}{|c|}{ No médio de lagartas ${ }^{1,2}$} & \multirow{2}{*}{\multicolumn{4}{|c|}{ Nota de dano $0^{1,2}$}} \\
\hline & & \multicolumn{3}{|c|}{ P. xylostella } & \multirow{2}{*}{$\frac{T . n i}{13 \mathrm{DA}^{\mathrm{a}} \mathrm{A}}$} & & & & \\
\hline & & $7 \mathrm{DA}^{\mathrm{a}} \mathrm{A}^{3}$ & $7 \mathrm{DA}^{\mathrm{a}} \mathrm{A}^{4}$ & $13 \mathrm{DA}^{\mathrm{a}} \mathrm{A}^{6}$ & & $7 \mathrm{DA}^{\mathrm{a}} \mathrm{A}$ & $7 \mathrm{DA}^{\mathrm{a}} \mathrm{A}$ & $6 \mathrm{DA}^{\mathrm{a}} \mathrm{A}^{5}$ & $13 \mathrm{DA}^{\mathrm{a}} \mathrm{A}$ \\
\hline 1. indoxacarb & 18 & $0,33 \mathrm{c}$ & $0,02 \mathrm{c}$ & $0,35 d$ & $0,00 \mathrm{~b}$ & $1,40 \mathrm{~b}$ & $0,20 \mathrm{c}$ & $2,15 c$ & $0,43 d$ \\
\hline 2. indoxacarb & 24 & $0,11 \mathrm{c}$ & $0,00 \mathrm{c}$ & $0,25 d$ & $0,00 \mathrm{~b}$ & $1,06 \mathrm{~b}$ & $0,03 \mathrm{c}$ & $1,18 \mathrm{~cd}$ & $0,00 \mathrm{~d}$ \\
\hline 3. indoxacarb & 30 & $0,09 \mathrm{c}$ & $0,10 \mathrm{c}$ & $0,25 d$ & $0,00 \mathrm{~b}$ & $0,69 \mathrm{~b}$ & $0,07 \mathrm{c}$ & $0,12 \mathrm{~d}$ & $0,00 \mathrm{~d}$ \\
\hline 4. indoxacarb & 36 & $0,14 \mathrm{c}$ & $0,05 \mathrm{c}$ & $0,05 d$ & $0,00 \mathrm{~b}$ & $0,55 \mathrm{~b}$ & $0,15 \mathrm{c}$ & $0,35 \mathrm{~cd}$ & $0,12 \mathrm{~d}$ \\
\hline 5. indoxacarb & 42 & $0,06 \mathrm{c}$ & $0,07 \mathrm{c}$ & $0,20 \mathrm{~d}$ & $0,00 \mathrm{~b}$ & $0,71 \mathrm{~b}$ & $0,25 \mathrm{c}$ & $0,07 \mathrm{~d}$ & $0,12 \mathrm{~d}$ \\
\hline 6. deltametrina & 6 & $2,57 a b$ & $1,32 a b$ & $5,00 \mathrm{~b}$ & $0,20 \mathrm{~b}$ & 7,57 a & $5,25 \mathrm{~b}$ & $13,85 a b$ & $13,00 \mathrm{~b}$ \\
\hline 7.esfenvalerate & 172 & $1,48 b$ & $1,15 b$ & $3,05 \mathrm{c}$ & $0,20 \mathrm{~b}$ & $6,53 \mathrm{a}$ & $3,39 \mathrm{~b}$ & $8,06 \mathrm{~b}$ & $8,62 \mathrm{c}$ \\
\hline 8. testemunha & - & $2,71 \mathrm{a}$ & $1,92 \mathrm{a}$ & $9,20 \mathrm{a}$ & $1,00 \mathrm{a}$ & $7,80 \mathrm{a}$ & $12,95 \mathrm{a}$ & $17,75 a$ & $19,50 \mathrm{a}$ \\
\hline $\mathrm{CV}(\%)$ & & 45,53 & 57,95 & 31,42 & 27,10 & 35,52 & 52,12 & 37,01 & 33,75 \\
\hline
\end{tabular}

${ }^{1 /}$ Dados originais;

${ }^{2 /}$ Médias seguidas de mesma letra na coluna não diferem entre si pelo teste de Tukey ao nível de 5\% de probabilidade. A análise estatística foi realizada com os dados transformados em $\sqrt{x+0,5}$

${ }^{3 /} 7 \mathrm{DA} 3^{\mathrm{a}} \mathrm{A}=$ sete dias após a $3^{\mathrm{a}}$ aplicação; ${ }^{4 /} 7 \mathrm{DA} 4^{\mathrm{a}} \mathrm{A}=$ sete dias após a $4^{\mathrm{a}}$ aplicação; ${ }^{5 /} 6 \mathrm{DA} 5^{\mathrm{a}} \mathrm{A}=$ seis dias após a $5^{\mathrm{a}}$ aplicação;

${ }^{6} 13 \mathrm{DA} 5^{\mathrm{a}} \mathrm{A}=$ treze dias após a $5^{\mathrm{a}}$ aplicação.

\section{Experimento III}

A eficiência do uso do indoxacarb no controle da broca-grande foi obtida em plantio de tomate estaqueado em Indianópolis, MG, adotando-se a mesma variedade e o mesmo espaçamento mencionados no experimento II. Do mesmo modo, adotou-se o delineamento em blocos casualizados com sete tratamentos e quatro repetições. A parcela também foi mantida com 20 plantas (duas linhas de dez plantas). As doses do inseticida indoxacarb foram as mesmas utilizadas no experimento II. Os demais tratamentos foram esfenvalerate na dose de $21,5 \mathrm{~g}$ i.a. $/ 100 \mathrm{~L}$, triflumuron na dose de $15,0 \mathrm{~g}$ i.a. $/ 100 \mathrm{~L}$ e a testemunha, sem aplicação de inseticida. Essas doses foram baseadas no consumo de $1.000 \mathrm{~L}$ de calda/ha. Foram realizadas quatro aplicações em intervalos semanais de $10 / 02$ a 10/03/97. A tecnologia de aplicação dos tratamentos foi idêntica à mencionada no experimento II.

As avaliações para a broca-grande foram realizadas um e sete dias após a última aplicação. Observou-se a presença de danos de $H$. zea em cinco frutos de cada uma das cinco plantas amostradas aleatoriamente na porção central de cada parcela, totalizando 25 frutos por parcela, e determinou-se a porcentagem de frutos sadios (não brocados) por parcela. O cálculo da eficiência de controle de cada tratamento utilizado, bem como os procedimentos estatísticos realizados foram os mesmos mencionados no experimento II.

\section{RESULTADOS E DISCUSSÃO}

Observa-se, a partir dos resultados obtidos com a testemunha, que a infestação da traça-das-crucíferas aumentou durante o período de condução do ensaio (Tabela 1). O tratamento à base de deltametrina (adotado como padrão) apresentou diferença estatística em relação à testemunha apenas na última avaliação (13 dias após o término das aplicações). A falha no controle observada com este produto está de acordo com resultados de França \& Medeiros (1998). Tal falha pode estar relacionada ao aumento dos alelos que conferem a resistência a esse produto na população de insetos (Castelo Branco \& Gatehouse, 1997), uma vez que se trata de um inseticida de uso amplamente difundido no controle de pragas de hortaliças.

As plantas tratadas com esfenvalerate, apesar de apresentarem um número médio de lagartas inferior à testemunha, não diferiram do tratamento à base de deltametrina nas avaliações realizadas sete dias após as $3^{\mathrm{a}} \mathrm{e} 4^{\mathrm{a}}$ aplicações. Todas as plantas tratadas com o produto indoxacarb apresentaram infestação de lagartas (traça-dascrucíferas e lagarta-mede-palmo) significativamente inferior àquela apresentada pela testemunha $(\mathrm{P}<0,05)$, não havendo, contudo, diferenças entre as doses testadas desse produto. Apesar da ocorrência da lagarta-mede-palmo ter sido restrita apenas à última avaliação, o produto indoxacarb nas doses testadas mostrou-se eficiente no controle desse inseto. Testes em situações de maior pressão dessa praga devem ser conduzidos para comprovar o efeito inseticida sobre $T$. ni. No entanto, Liu et al. (2002) também verificaram que o inseticida indoxacarb foi eficiente no controle da lagarta-mede-palmo em repolho; porém utilizando dose maior (50 g i.a./ha).

Em relação ao dano causado pela traça-das-crucíferas (Tabela 1), nota-se que todas as doses do produto indoxacarb foram eficientes para reduzir o nível de desfolha causado pela praga. Ainda, esses tratamentos foram mais eficientes do que os produtos deltametrina e esfenvalerate, que apresentaram comportamento semelhante entre si. Em duas avaliações, sete dias após a $3^{\mathrm{a}}$ aplicação e seis dias após a $5^{\mathrm{a}}$ 
Tabela 2. Avaliação da eficiência do indoxacarb em diferentes doses no controle da broca-pequena, Neoleucinodes elegantalis (Lepidoptera: Pyralidae). Mogi-Guaçu (SP), FCAV/UNESP, 1997.

\begin{tabular}{|c|c|c|c|c|c|}
\hline \multirow{3}{*}{ Tratamento } & \multirow{3}{*}{$\begin{array}{c}\text { Dose } \\
\text { (g i.a./100 L) }\end{array}$} & \multicolumn{4}{|c|}{ Avaliações $^{1,2}$} \\
\hline & & \multicolumn{2}{|c|}{$5 \mathrm{DA}^{\mathrm{a}} \mathrm{AP}^{3}$} & \multicolumn{2}{|c|}{ 4 DA9 ${ }^{\mathrm{a}} \mathrm{AP}^{4}$} \\
\hline & & $\begin{array}{c}\text { Frutos } \\
\text { brocados (\%) }\end{array}$ & $\begin{array}{l}\text { Eficiência de } \\
\text { controle (\%) }\end{array}$ & $\begin{array}{c}\text { Frutos } \\
\text { brocados (\%) }\end{array}$ & $\begin{array}{l}\text { Eficiência de } \\
\text { controle (\%) }\end{array}$ \\
\hline 1. indoxacarb & 2,40 & $9,96 \mathrm{a}$ & 30,05 & $0,75 \mathrm{~b}$ & 96,08 \\
\hline 2. indoxacarb & 3,60 & $5,96 a$ & 61,30 & $2,73 \mathrm{~b}$ & 86,32 \\
\hline 3. indoxacarb & 4,80 & $8,79 a$ & 36,16 & $2,87 \mathrm{~b}$ & 84,84 \\
\hline 4. indoxacarb & 6,00 & $4,96 a$ & 42,55 & $1,40 \mathrm{~b}$ & 93,23 \\
\hline 5. esfenvalerate & 1,75 & $9,67 \mathrm{a}$ & 12,08 & $4,60 \mathrm{~b}$ & 73,80 \\
\hline 6. metomil & 21,50 & $5,96 a$ & 36,30 & $0,97 \mathrm{~b}$ & 95,32 \\
\hline 7. testemunha & - & $10,23 a$ & - & $19,78 \mathrm{a}$ & - \\
\hline CV (\%) & & 40,37 & & 41,26 & \\
\hline
\end{tabular}

${ }^{1 /}$ Dados originais (em porcentagem);

${ }^{2 /}$ Médias seguidas da mesma letra na coluna não diferem pelo teste de Tukey ao nível de $5 \%$ de probabilidade. A análise estatística foi realizada com os dados transformados em $\operatorname{arc} \operatorname{sen} \sqrt{\%}$

${ }^{3 /} 5 \mathrm{DA} 5^{\mathrm{a}} \mathrm{AP}=$ cinco dias após a $5^{\mathrm{a}}$ aplicação;

4/ $4 \mathrm{DA} 9^{\mathrm{a}} \mathrm{AP}=$ quatro dias após a $9^{\mathrm{a}}$ aplicação.

Tabela 3. Avaliação da eficiência do indoxacarb em diferentes doses no controle da broca-grande, Helicoverpa zea (Lepidoptera: Noctuidae). Indianópolis (MG), FCAV/UNESP, 1997.

\begin{tabular}{|c|c|c|c|c|c|}
\hline \multirow{3}{*}{ Tratamento } & \multirow{3}{*}{$\begin{array}{c}\text { Dose } \\
\text { (g i.a./100 L) }\end{array}$} & \multicolumn{4}{|c|}{ Avaliações $^{1,2}$} \\
\hline & & \multicolumn{2}{|c|}{1 DAUA $^{3}$} & \multicolumn{2}{|c|}{7 DAUA $^{4}$} \\
\hline & & $\begin{array}{c}\text { Frutos sadios } \\
\text { (\%) }\end{array}$ & $\begin{array}{l}\text { Eficiência de } \\
\text { controle (\%) }\end{array}$ & $\begin{array}{c}\text { Frutos sadios } \\
(\%)\end{array}$ & $\begin{array}{l}\text { Eficiência de } \\
\text { controle (\%) }\end{array}$ \\
\hline 1. indoxacarb & 2,40 & 98,39 a & 87,78 & 95,50 a & 78,70 \\
\hline 2. indoxacarb & 3,60 & 98,17 a & 85,73 & 97,82 a & 87,07 \\
\hline 3. indoxacarb & 4,80 & 99,20 a & 89,61 & 98,28 a & 90,66 \\
\hline 4. indoxacarb & 6,00 & 98,85 a & 88,98 & 98,72 a & 91,11 \\
\hline 5.esfenvalerate & 21,50 & 98,72 a & 95,30 & 97,12 a & 84,14 \\
\hline 6. triflumuron & 15,00 & 98,32 a & 84,37 & 98,70 a & 92,45 \\
\hline 7. testemunha & - & $79,12 b$ & - & $80,20 \mathrm{~b}$ & - \\
\hline $\mathrm{CV}(\%)$ & & 6,96 & & 5,23 & \\
\hline
\end{tabular}

aplicação, as plantas tratadas com deltametrina apresentaram níveis de dano que não diferiram dos da testemunha. Esse resultado traduz a falha no controle do inseto com deltametrina pois as plantas neste tratamento foram tão atacadas pela praga quanto as da testemunha que não receberam qualquer tratamento com inseticida. $\mathrm{O}$ aumento da infestação da traças-das-crucíferas durante o experimento (Tabela 1) é acom- panhado pela elevação das notas de dano causado pela praga na testemunha. Pode-se observar ainda que todos os tratamentos à base de indoxacarb apresentaram nota média de dano próxima a zero, ou seja, as plantas praticamente não apresentaram dano. Por outro lado, as plantas tratadas com deltametrina $\mathrm{e}$ esfenvalerate assim como as da testemunha, apresentaram dano médio próximo ou superior a 10 , principalmente nas duas últimas avaliações, ou seja, folhas laterais furadas e algumas cabeças já apresentando furos. Assim, com base nos resultados de infestação das pragas e dos danos causados por $P$. xylostella, a dose de $18 \mathrm{~g}$ i.a./ha pode ser utilizada no controle da traça-dascrucíferas e da lagarta-mede-palmo.

Na primeira avaliação, realizada cinco dias após a $5^{\text {a }}$ aplicação, não se observou diferença estatística $(\mathrm{P}>0,05)$ 
entre os tratamentos com relação à porcentagem de frutos danificados pela broca-pequena (Tabela 2). Entretanto, na segunda avaliação, todos os tratamentos inseticidas apresentaram diferença significativa em relação à testemunha $(\mathrm{P}<0,05)$, com relação a esse parâmetro. Observa-se que houve aumento na infestação pela praga, comprovado pelo aumento na porcentagem de frutos brocados na testemunha. Na última avaliação, com exceção do tratamento à base de esfenvalerate, todos os tratamentos inseticidas apresentaram eficiência superior a $80 \%$ na redução dos danos causados pela praga, sendo, portanto, eficientes no seu controle. Assim, o produto indoxacarb pode ser utilizado para o controle da broca-pequena do tomateiro, podendo ser recomendada a menor dose avaliada (2,4 g i.a./100 L), que também demonstrou eficiência no controle da praga.

Com exceção apenas do produto indoxacarb aplicado na dose de 2,4 $\mathrm{g}$ i.a./100 L, na avaliação realizada sete dias após a última aplicação, todos os demais produtos e doses testadas apresentaram eficiência de controle superior a $80 \%$ para $H$. zea (Tabela 3 ). Não foi observado qualquer sintoma de fitotoxicidade causada pelo produto indoxacarb nas culturas de repolho e tomate. Assim, de acordo com os resul- tados obtidos, pode-se concluir que o inseticida indoxacarb é eficiente no controle das pragas de hortaliças alvos deste trabalho nas doses testadas, podendo ser utilizado no controle químico dessas pragas.

\section{LITERATURA CITADA}

ABBOTT, W.S. A method of computing the effectiveness of an insecticide. Journal of Economic Entomology. v. 18, p. 265-267, 1925. AGRIANUAL, São Paulo, FNP, 94 p., 2000.

BARBOSA, S.; FRANÇA, F.H. As pragas do tomateiro e seu controle. Informe Agropecuário, Belo Horizonte, v. 6, p. 37-40, 1980.

CASTELO BRANCO, M.; GUIMARÃES, A.L. Controle da traça-das-crucíferas em repolho, 1989. Horticultura Brasileira, Brasília, v. 8, n. 1, p. 24 25,1990.

CASTELO BRANCO, M.; GATEHOUSE, A.G. Insecticide resistance in Plutella xylostella (L.) (Lepidoptera: Yponomeutidae) in Federal District, Brazil. Anais da Sociedade Entomológica do Brasil, Londrina, v. 26, p. 75-79, 1997.

FRANÇA, F.H.; MEDEIROS, M.A. Imapacto da combinação de inseticidas sobre a produção de repolho e parasitóides associados com a traça-dascrucíferas. Horticultura Brasileira, Brasília, v. 16, n. 2, p. 132-135, 1998.

HARDER, H.H.; RILEY, S.L.; McCANN, S.F. DPX-MP062: A novel broad spectrum, environmentally soft, insect control compound Brighton Crop Protection Conference - Pests \& Diseases, 1996.

LIU, T.X.; SPARKS JR., A.N.; CHEN, W.; LIANG G.M.; BRISTER, C. Toxicity, persistence, and efficacy of indoxacarb on cabbage looper (Lepidoptera: Noctuidae) on cabbage. Journal of Economic Entomology, v. 95, n. 2, p. 360-367, 2002.
McCANN, S.F.; ANNIS, G.D.; SHAPIRO, R.; PIOTROWSKI, D.W.; LAWHM, G.P.; LONG, J.K.; LEE, K.C.; HUGHES, M.M.; MYERS, B.J.; GRISWOLD, S.M. The discovery of indoxacarb: oxadiazines as a new class of pyrazoline-type insecticides. Pest management Science, v. 57, n. 2, p. 153-164, 2001.

MONNERAT, R.G.; BORDAT, D.; CASTELO BRANCO, M.; FRANÇA, F.H. Efeito de Bacillus thuringiensis Berliner e inseticidas químicos sobre a traça-das-crucíferas, Plutella xylostella (Lepidoptera: Yponomeutidae) e seus parasitóides. Anais da Sociedade Entomológica do Brasil, Londrina, v. 29, n. 3, p. 723-730, 2000.

NAKANO, O. As pragas das hortaliças: seu controle e o selo verde. Horticultura Brasileira, Brasília, v. 17, n. 1, p. 04-05, 1999.

PLUSCHKELL, U.; HOROWITZ, A.R.; WEINTRAUB, P.G.; ISHAAYA, I. DPX-MP062 - a potent compound for controlling the Egyptian cotton leafworm Spodoptera littoralis (Boisd.). Pesticide Science, v. 54, n. 2, p. 85-90, 1998.

REIS, P.R.; SOUZA, J.C. Controle da broca-pequena, Neoleucinodes elegantalis (Guenée, 1854) (Lepidoptera, Pyralidae), com inseticidas fisiológicos em tomateiro estaqueado. Anais da Sociedade Entomológica do Brasil, Londrina, v. 25, n. 1, p. 65-69, 1996.

WING, K.D.; SCHNEE, M.; SACHER, M.; CONNAIR, M. A novel oxidiazine insecticide is bioactivated in lepidopteran larvae. Archives of insect biochemistry and physiology, v. 37, p. 91103, 1998.

WING, K.D.; SACHER, M.; KAGAYA, Y.; TSURUBUCHI, Y.; MULDERIG, L.; CONNAIR, M.; SCHNEE, M. Bioactivation and mode of action of the oxidiazine indoxacarb in insects. Crop Protection, v. 19 n. 8/10, p. 537-545, 2000. 